\title{
Glory oscillations in photoionization microscopy: Connection with electron dynamics and Stark spectral structures in the continuum
}

\author{
P. Kalaitzis,,${ }^{1, *}$ S. Danakas,${ }^{1}$ K. Ferentinou, ${ }^{1}$ F. Lépine, ${ }^{2}$ C. Bordas,${ }^{2}$ and S. Cohen $\oplus^{1, \dagger}$ \\ ${ }^{1}$ Atomic and Molecular Physics Laboratory, Physics Department, University of Ioannina, 45110 Ioannina, Greece \\ ${ }^{2}$ Université de Lyon, CNRS, UMR5306, Institut Lumière Matière, 69622 Villeurbanne, France
}

(Received 15 June 2020; accepted 6 August 2020; published 1 September 2020)

\begin{abstract}
In classical scattering theory, the term "glory scattering" implies the divergence of the classical differential cross section that occurs as soon as the deflection function goes through zero for a nonzero value of the impact parameter. This critical effect also occurs in slow photoelectron imaging where near-threshold atomic photoionization is performed in the presence of an external static electric field. In this case, glory scattering manifests itself by the appearance of an intense peak at the center of the photoelectron momentum distribution. In the present work we examine the magnitude variation of this central peak as a function of electron energy. We experimentally study near-threshold two-photon ionization of ground state magnesium atoms, below as well as above the field-free ionization limit. It is found that, apart from its behavior of classical origin, the glory signal additionally exhibits strong oscillations and beating effects over the full spectral range of the recordings. Of particular interest are its oscillations above the zero-field limit, many aspects of which are expected to be independent of the atomic target. Our results are analyzed with the help of classical, semiclassical, and quantum mechanical calculations devoted to the hydrogenic Stark effect. It is theoretically found that these continuum glory oscillations are related to the resonantlike Stark structures appearing under certain conditions in the total photoionization cross section and implying energy quantization in the continuum. The striking outcome of the present study, however, is that both theory and experiment clearly support the connection between the energyand static field-dependent periodicity of glory oscillations with the classical dynamics of electron motion. In particular, it is shown that the Fourier transform of the glory signal provides information on the differences between the origin-to-detector times of flight corresponding to specific pairs of classical electron trajectories.
\end{abstract}

DOI: 10.1103/PhysRevA.102.033101

\section{INTRODUCTION}

The interaction of an atom with an external static electric field is a fundamental problem in quantum physics. Due to the presence of the field, the bound states of the atom are, strictly speaking, converted to quasibound ones (resonances), while its ionization threshold is lowered with respect to its zero-field value (set at zero energy, $E=0$ ). Between the field-induced and the zero-field limits continuum states and resonances coexist. Furthermore, even for $E>0$ resonantlike structures appear in the total ionization cross section. These static-fieldinduced structures imply energy quantization in the continuum and have no counterpart when the field is turned-off. The above specific features explain the rich phenomenology associated with near-threshold atomic photoionization under the presence of the field. This phenomenology is nowadays suitably studied by photoionization microscopy (PM) [1], the term denoting a high-resolution experimental technique where the two-dimensional flux of slow (meV) photoelectrons is imaged by a position sensitive detector (PSD). In an attempt towards the categorization and classification of the above-

*Present address: Institute for Molecules and Materials, Radboud University, Heijendaalseweg 135, 6525 AJ Nijmegen, The Netherlands

†Corresponding author: scohen@uoi.gr mentioned phenomenology, photoionization was described in terms of classical particle scattering in conjunction with the source-to-detector trajectories followed by the electrons under the action of the field [1-6]. These studies revealed that the classical differential photoionization cross section exhibits divergences leading to critical scattering effects such as the so-called rainbow and glory scattering [7-9]. Rainbow scattering is responsible for the appearance of concentric structures of different radii in the images, despite the fact that these images are formed by slow single-energy electrons. This is in contrast to the usual hypothesis made in standard (eV range) electron imaging spectroscopy, where a one-to-one correspondence is assumed between the number of image rings and the number of electron groups of different energy. Moreover, as long as a given photoionization scenario leads to the production of electrons with zero projection of the electron's orbital angular momentum on the static field axis, glory scattering is responsible for the appearance of a high intensity central peak on the images. As mentioned in Ref. [4], this peak might occasionally be erroneously interpreted as originating from zero energy electrons in the analysis of threshold photoelectron spectroscopy data.

The above signatures of classical critical effects in slow photoelectron imaging were experimentally observed at several instances [6,10-15]. They are also clearly imprinted in the presently acquired images obtained by near-threshold twophoton ionization of ground state $\mathrm{Mg}$ atoms in the presence of 


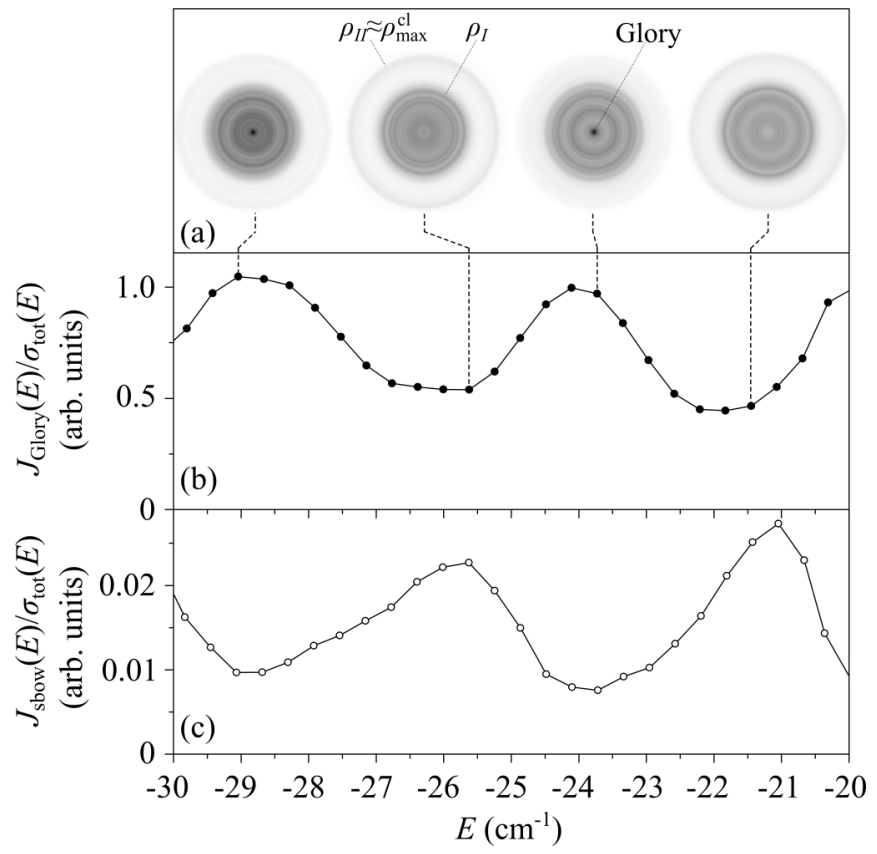

FIG. 1. (a) Experimental photoionization microscopy images $J_{v_{\text {det }}}(\rho)$ obtained by near-threshold two-photon ionization of ground state $\mathrm{Mg}$ atoms in an external static electric field of strength $F=$ $680 \pm 10 \mathrm{~V} / \mathrm{cm}$. The gray scale is the same for all images and it is stretched from zero (white) to $100 \%$ (black). The energy scale at the bottom of the figure refers to the zero-field limit. Linear laser beam polarization parallel to the field ( $m=0$ final Stark states, explaining the absence of angular dependence in the images). The glory electron signal at the center of the image and the radii corresponding to primary $\left(\rho_{I}\right)$ and secondary $\left(\rho_{I I}\right)$ rainbow scattering are noted in the two images. In fact, the radius $\rho_{I I}$ is practically identical to the maximum classical radius $\rho_{\max }^{\mathrm{cl}}$. The intensity variation with energy of the outer rainbow and the glory signal is obvious. (b) Detailed energy evolution of the glory intensity, where, in addition to the data extracted from the images shown in (a), the plot includes all measurements within the given interval. For eliminating any dependence on the variation of the laser pulse energy during the recordings, the glory signal is scaled to the total one, the latter obtained by integrating over the whole surface of the detector. This total signal is proportional to the total ionization cross section $\sigma_{\text {tot }}$. (c) Detailed energy evolution of the signal of the outer rainbow ring $\left(\rho_{I I}\right)$ scaled to the total one. At this energy range bright rainbow signals occur when the glory intensity exhibits minima and vice versa. The intensity $y$ axis units are arbitrary but common to (b) and (c).

a static electric field. A sample of these images is given in Fig. 1(a). In fact, because of the high spatial resolution of our photoionization microscope, the images also show important quantum interference and beating phenomena. Additionally, due to the adequate spectral resolution of the experiment, quite noticeable among these phenomena is the intensity modulation of the glory signal as a function of the energy. This modulation is more clearly observed in the plot of Fig. 1(b) which includes the full set of measurements within the corresponding fraction of the "glory spectrum." It is also interesting to notice in the images of Fig. 1(a) and the "spectrum" of Fig. 1(c) the similar intensity modulation of the outer (rainbow) image ring, where at this energy range bright rainbow signals occur when the glory intensity exhibits minima and vice versa.

The thorough investigation of these oscillations and their implications is the subject of the present work. Our intention is to go beyond the classical description of critical scattering phenomena given in Ref. [4] and consider these additional features whose interpretation requires at least a semiclassical $[1,6,10]$ or, better, a quantum mechanical description. To this purpose we employ hydrogenic calculations based on the formulation of Ref. [16] and experimental results regarding the nonhydrogenic $\mathrm{Mg}$ atom. Such a comparison is important because it allows for the distinction of those features which are of global nature, from those that are specific to the examined atom and excitation scheme. However, due to the (occasionally abrupt) variation of the bow radius with energy, the rainbow signals probe interferences occurring at different points of the detector. This fact makes them conceptually more difficult to interpret and they are only partially discussed in connection with the glory ones. Therefore, the present article focuses mainly on the glory effect, because the signal at the image center is easy to define and record, its relative intensity near threshold dominates over all other image features and its observation does not require any particular performance from the imaging spectrometer. It is shown that the oscillations of this signal bear a connection with the aforementioned positive energy static-field-induced resonant structures. Furthermore, and even more importantly, it is undoubtedly proved theoretically and verified experimentally that glory oscillations in the continuum contain time-domain information. Specifically, it is found that the Fourier transform of the glory signal leads to the knowledge of the differences between the times of flight towards the detector that correspond to specific pairs of classical electron trajectories. From this point of view, our observations share some similarities with a subject of active present discussion within an apparently different context, i.e., within the search of alternative approaches towards the inference of electron dynamics from spatial electron interference effects into which they are encoded [6,17], particularly when dealing with the interaction of atomic and molecular systems with ultrashort laser pulses.

The rest of the paper is organized as follows: In Sec. II we make a brief successive presentation of the classical, semiclassical, and quantum theoretical frameworks of the Stark effect in the vicinity of the ionization threshold. This section also includes a first comparison among theoretical results on critical scattering effects, as computed by all of the above three approaches. Section III gives a short description of the experimental setup and procedure. Experimental results are presented and discussed in connection with the theoretical ones in Sec. IV. Finally, in Sec. V we give our concluding remarks and discuss directions for further work.

\section{THEORY}

\section{A. Classical and semiclassical description of slow photoelectron imaging and its connection to critical scattering phenomena}

Critical phenomena, such as the glory signal, can be predicted by the classical scattering theory. Therefore, it would be helpful for the reader to begin with a brief reminder of the 
connection of this theory with the classical features of slow photoelectron imaging [1-6,10-15].

Let us first consider the classical electron trajectories in the combined presence of an attractive Coulomb center and a homogeneous static electric field $\mathbf{F}=F \mathbf{z}$. The classical equations of motion for the electron are separable in parabolic (or "semiparabolic" as they are also labeled quite frequently) coordinates [18,19] $\chi=(r+z)^{1 / 2} \geqslant 0, v=(r-z)^{1 / 2} \geqslant 0$, and $\varphi=\tan ^{-1}(y / x)$, with $r=\left(x^{2}+y^{2}+z^{2}\right)^{1 / 2}$ being the distance of the electron from the residual structureless ion of charge $Z$ (for hydrogen atom $Z=1$ ). In particular, we are interested in those trajectories that lead to ionization, i.e., to the ejection of the electron towards the PSD whose plane is perpendicular to the $z$ axis and positioned at $z=z_{\mathrm{det}}=$ $-v_{\mathrm{det}}^{2} / 2<0$. Therefore, the energy range of interest lies above the classical saddle-point energy [20], $E_{s p}^{\mathrm{cl}}=-2(Z F)^{1 / 2}[$ in atomic units (a.u.) $\left.\hbar=e=m_{e}=1\right]$, i.e., the classical field ionization threshold, which is located below the zero-field ionization limit (at energy $E=0$ ). For convenience, in what follows, we employ alternatively to the energy of the system $E$, the reduced energy variable

$$
\varepsilon \equiv \frac{E}{\left|E_{s p}^{\mathrm{cl}}\right|}
$$

Furthermore, since in our case the appearance of the glory effect is associated solely with zero projection of the orbital angular momentum on the field $z$ axis [4], the present discussion is restricted to this situation leading to a planar electron motion (where the coordinate $\varphi$ is time independent and equal to an initial value $\varphi_{\mathrm{o}}$ which can be conveniently set equal to zero, $\varphi=\varphi_{\mathrm{o}}=0$ ). It turns out that the $\chi$ motion (perpendicularly to the field direction when $z$ tends to $-\infty$ ) is bound and periodic. Along the $v$ coordinate (i.e., along the field direction when $z$ tends to $-\infty$ ) the electron escapes and reaches the detector at $v=v_{\text {det }}$. For a given energy $E \geqslant E_{s p}^{\mathrm{cl}}$ $(\varepsilon \geqslant-1)$, a classification of the different types of trajectories may be accomplished via two parameters: First, by the electron's initial ejection angle $\beta$ with respect to the external electric field, where $\beta=0$ denotes uphill ejection $(+z)$ and $\beta=\pi$ downhill ejection $(-z)$ towards the PSD. Second, by the number $Q(\varepsilon, \beta)$ of half- $\chi$ oscillations performed until the electron reaches the detector. The analytical expression for $Q$ for $\left|z_{\text {det }}\right|$ (and $v_{\text {det }}$ ) $\rightarrow \infty$ is given in the Appendix. The integer part of $Q$ provides the number of intersections between the trajectory and $z$ axis. Thus, values $Q>1$ correspond to complicated (hereafter called indirect) electron trajectories which intersect the negative $z$ axis at least once and appear for $\varepsilon \geqslant-1$. The simpler $0<Q<1$ range characterizes quasiparabolic source-to-detector (direct) trajectories that do not intersect the $z$ axis. The distinction between direct and indirect trajectories is achieved by solving the equation $Q\left(\varepsilon, \beta_{0}\right)=1$ for the critical angle $\beta_{0} \leqslant \pi$. This equation has no meaningful solution for $\varepsilon<\varepsilon_{\mathrm{dir}} \approx-0.775$ [11] and, therefore, direct trajectories exist only for $\varepsilon \geqslant \varepsilon_{\mathrm{dir}}$, with $\beta_{0}\left(\varepsilon_{\mathrm{dir}}\right)=\pi$. Finally, for $-1 \leqslant \varepsilon \leqslant 0$ any type of trajectory corresponds to angles $\beta \geqslant \beta_{\mathrm{c}} \equiv \arcsin [|\varepsilon|]$ (for $\beta<\beta_{\mathrm{c}}$ the electron does not escape from the atom), while $\beta_{\mathrm{c}}=0$ for $\varepsilon \geqslant 0$.

The distribution of photoelectron impacts on the PSD gives rise to images that may be computed by means of the classical trajectories described above. For an isotropic electron source $[3,11]$ and for the planar, $\varphi$-independent, motion discussed here, it suffices to calculate the distribution of electron impacts $R$ as a function of the impact radius $\rho=\chi v_{\text {det }}$ on the detector. From the perspective of classical particle scattering photoionization is treated as a half-collision process $[4,5]$ and the distribution $R(\rho)$ corresponds to the classical differential ionization cross section. The latter may be written as

$$
R(\rho) \equiv \frac{d \sigma}{d A}(\rho)=\sum_{j} \frac{\sin \beta_{j}}{\rho}\left|\frac{d \rho}{d \beta_{j}}\right|^{-1},
$$

where $d A=2 \pi \rho d \rho$ is the elementary detector surface and the summation runs over all ejection angles $\beta_{j}$ leading to the same radius $\rho$. Equation (2) bears a striking formal similarity with the classical scattering differential cross section for a particle scattered by a central potential [7]. By regarding the impact radius on the detector as a generalized scattering deflection function, Eq. (2) shows that critical phenomena (i.e., singularities) are expected whenever $\rho(\beta)$ shows an extremum or whenever it goes through zero while $\sin \beta \neq 0$. By analogy with atmospheric optics the first type of singularity is named as rainbow scattering and the second one as glory scattering, respectively [7-9]. As the example of Fig. 2(a) shows, within the interval $\left[\beta_{\mathrm{c}}, \pi\right]$, this function exhibits several maxima and several zeros. The maxima of $\rho(\beta)$ give rise to rainbow scattering. For $\varepsilon \geqslant \varepsilon_{\text {dir }}$ there is a single maximum $\rho_{I}$ within the interval $\left[\beta_{0}, \pi\right]$, the so-called primary rainbow radius stemming from the direct trajectories. Generally speaking, the primary bow is more intense in the photoelectron image than all other secondary bows stemming from the maxima $\rho_{I I}>\rho_{I}$, $\rho_{I I I}>\rho_{I I}$, etc. that occur within the $\left[\beta_{\mathrm{c}}, \beta_{0}\right]$ interval. All these maxima are practically indistinguishable from $\rho_{I I}$, which, in turn, is almost identical to the analytically known [3-5] maximum radius of impact $\rho_{\max }^{\mathrm{cl}}$. The existence of primary and secondary maxima leads to a particular characteristic of slow photoelectron imaging, i.e., to the appearance of two concentric structures in the recorded images with radii $\rho_{I}$ and $\rho_{I I}$ [see Fig. 1(a)]. These structures progressively merge together at high positive energy. As for the zeros of $\rho(\beta)$, they occur at $\beta=\pi$ as well as at those angles $\beta_{k}$ that correspond to integer values of $Q\left(\varepsilon, \beta_{k}\right)=k+1, k=0,1,2, \ldots$ [see Fig. 2(b)]. For $\beta_{k} \neq 0$ and $\beta_{k} \neq \pi$ these zeros give rise to glory scattering, which is responsible for the appearance of a high intensity central peak in the photoelectron images. Experimentally the glory peak is generally found to be more intense than any bow. Figure 3(a) shows a classical calculation of the evolution of the glory intensity with energy. As already discussed in Ref. [4], the classical "glory spectrum" is characterized by two peaks, one located at the saddle-point energy $(\varepsilon=-1)$ and the second brighter one at $\varepsilon \approx \varepsilon_{\text {dir }}$. Apart from these peaks the magnitude of the glory signal decreases smoothly with increasing reduced energy $\varepsilon$.

Finally, for getting a smoother connection with the following quantum description, Fig. 3(a) also shows for comparison the semiclassically calculated energy evolution of the glory signal. The semiclassical theory of photoionization microscopy has been abundantly documented in the past $[1,2,11,12,21]$. Briefly, one first computes the phase $S$ accumulated along each classical electron trajectory from the 

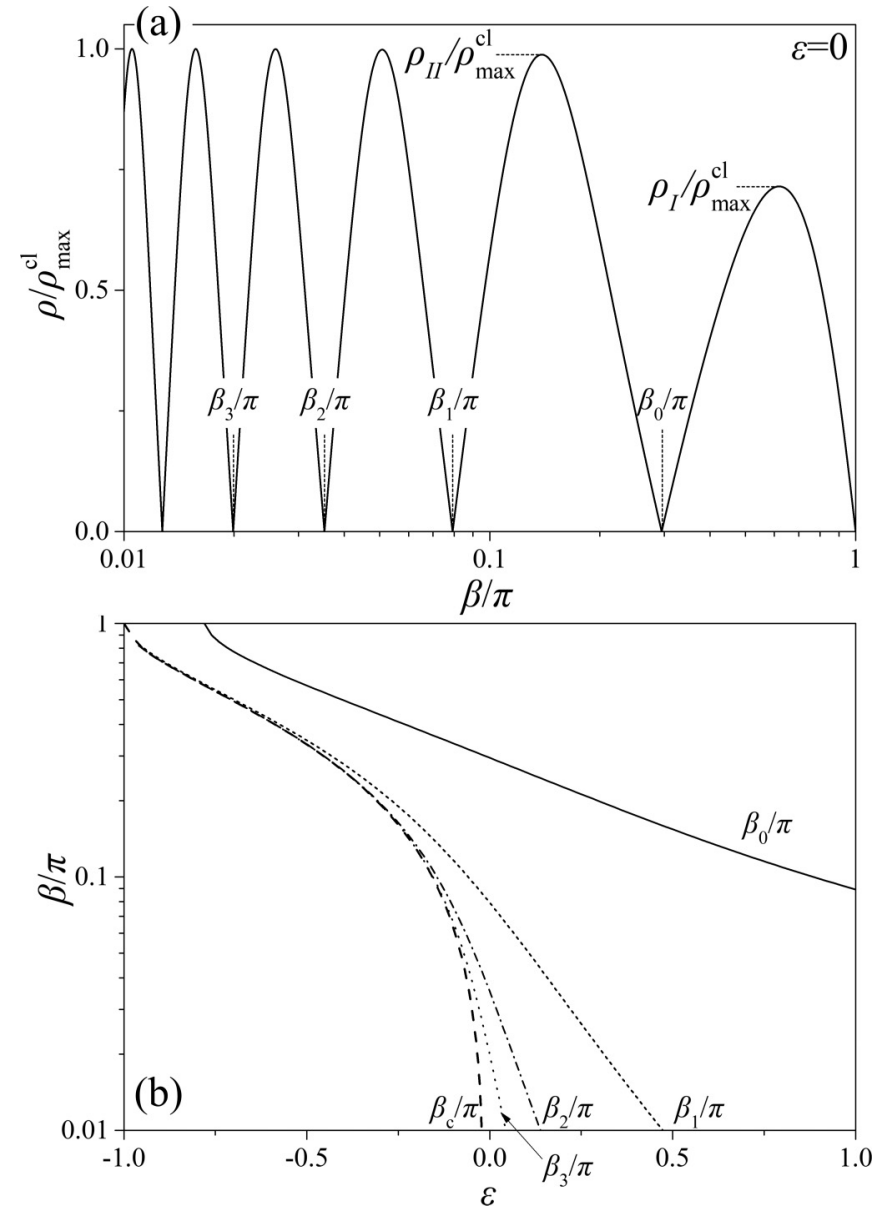

FIG. 2. (a) Classically calculated impact radius on the detector $\rho$ as a function of the ejection angle $\beta$ at $E=\varepsilon=0$. It is assumed that the detector is placed at infinity. The impact radius is scaled to the maximum radius of impact $\rho_{\max }^{\mathrm{cl}}(\varepsilon=0)$ [3-5]. Two maxima of $\rho(\beta)$ are marked (out of their infinite number), corresponding to the primary bow $\left(\rho_{I}\right)$ and first secondary bow $\left(\rho_{I I}\right)$, respectively. The number of zeros of $\rho(\beta)$ is also infinite. These zeros occur at $\beta=\pi$ and at the angles $\beta=\beta_{k}, k=0,1,2 \ldots,\left(\beta_{0}-\beta_{3}\right.$ are noted in the graph) obtained by solving the equation $Q\left(\varepsilon, \beta_{k}\right)=k+1$, where the function $Q(\varepsilon, \beta)$ is defined in the text and in the Appendix. Note the logarithmic $x$ scale, chosen in order to emphasize the large magnitude difference between the angles $\beta_{k}$ as $k$ increases. (b) Evolution of the critical angle $\beta_{\mathrm{c}}$ and the angles $\beta_{0}-\beta_{3}$ with reduced energy. The angle $\beta_{0}$ is defined for $\varepsilon \geqslant \varepsilon_{\text {dir }} \approx-0.775$, while for $k>0$ angles $\beta_{k}$ are defined for energies practically coinciding with $\varepsilon=-1$, just like $\beta_{\mathrm{c}}$ (which is zero for $\varepsilon \geqslant 0$ ). The logarithmic $y$ scale is chosen for the same reasons as in (a).

source to a given point of the detector. Subsequently, this phase provides the contribution of that trajectory to the final electron wave function [11],

$$
\Psi^{\mathrm{SC}}(\rho)=\sum_{j} \sqrt{\frac{\sin \beta_{j}}{\rho}\left|\frac{d \rho}{d \beta_{j}}\right|^{-1}} c_{j} e^{i S_{j}(\rho)},
$$

where the index $j$ is defined as in Eq. (2) and where the weight $c_{j}$ of each contribution is introduced. The radial distribution $R(\rho)$ is proportional to $\left|\Psi^{\mathrm{SC}}\right|^{2}$. In the semiclassical calculation

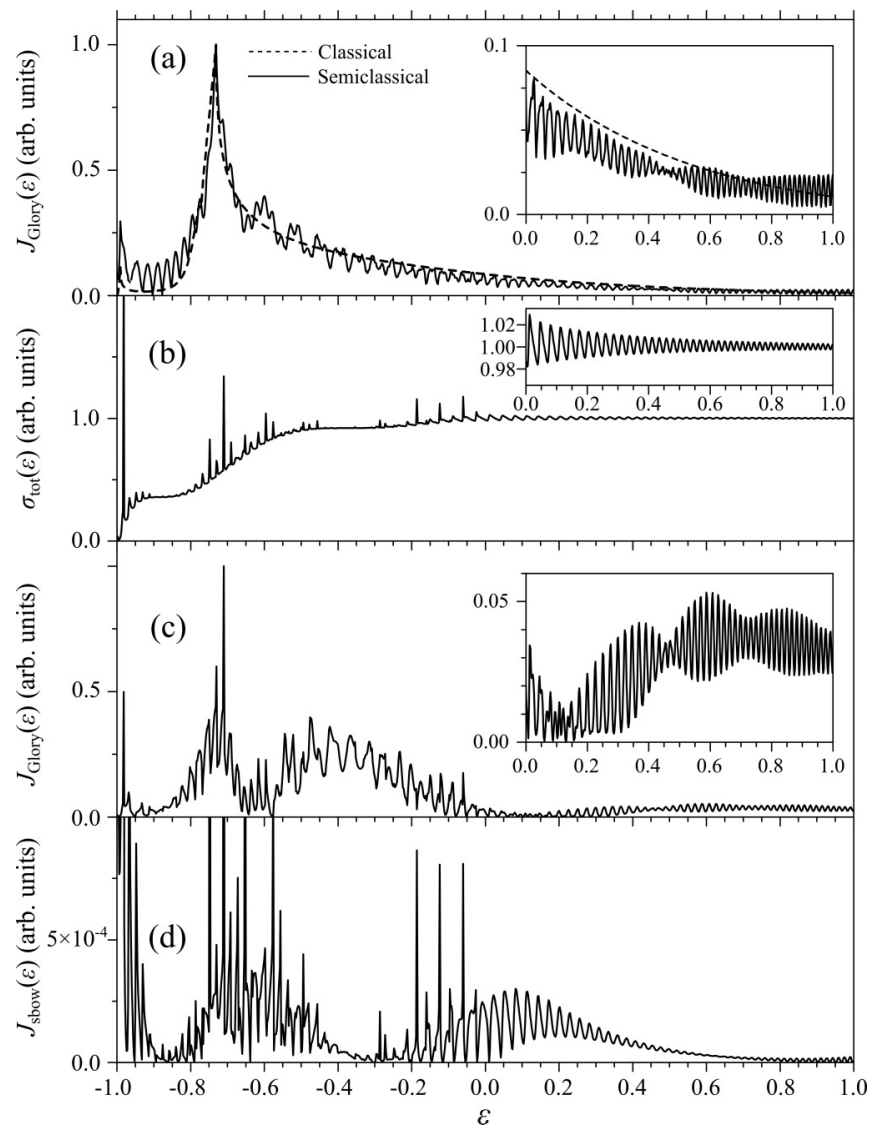

FIG. 3. (a) Energy dependence of the classical and semiclassical glory signals for a field strength $F=680 \mathrm{~V} / \mathrm{cm}$ in the case of the planar motion. The signals are computed under the assumption of an isotropic initial photoelectron emission and by integrating the corresponding radial distributions [Eq. (2) and squared modulus of Eq. (3), respectively] over a radius equal to $1 \%$ of $\rho_{\max }^{\mathrm{cl}}(\varepsilon=0)$. Both curves exhibit local maxima at $\varepsilon=-1$ (onset of indirect trajectories) and at $\varepsilon=\varepsilon_{\mathrm{dir}} \approx-0.775$ (onset of direct trajectories). The semiclassical curve additionally shows oscillations and beating effects. (b) Quantum mechanical total ionization cross section [Eq. (9)] for the same field as above and for two-photon excitation of $m=0$ final Stark states out of the hydrogenic ground state. The spectrum shows both quasibound states $(\varepsilon<0)$ and static-field-induced ones $(\varepsilon>0)$. (c) The corresponding glory signal [Eq. (10) computed with $v_{\text {det }}=$ 2000 atomic units, i.e., $\left.\left|z_{\operatorname{det}}\right| \approx 106 \mu \mathrm{m}\right]$ and (d) the corresponding secondary bow signal, defined here as $J_{\text {sbow }} \equiv J_{v_{\text {det }}}\left(\rho_{I I}\right)$ where $\rho_{I I}$ is approximated by the smooth expression for $\rho_{\max }^{\mathrm{cl}}$, the latter scaled to the outermost inflection point of the radial distribution $R(\rho)$ at $\varepsilon=0$. The intensity $y$-axis units are arbitrary but common to (c) and (d). The insets in (a)-(c) display magnified views of the respective signals at positive energies.

of Fig. 3(a) the most frequent choice of equal weights $c_{j}$ is employed. Apart from the gross energy dependence, which is common with its classical counterpart, the semiclassical curve is additionally characterized by oscillations of appreciable amplitude and intense beating effects [see inset of Fig. 3(a)]. The "carrier frequency" of these oscillations appears to be field strength and energy dependent. 


\section{B. Quantum mechanical Coulomb-Stark problem: Current probability density and the glory signal}

Let us now recall the essential elements of the quantum mechanical theory of the Stark effect, by which one is led to the calculation of PM images, total photoionization cross sections, and glory and bow signals [16]. Like the classical equations of motion, the Schrödinger equation for the hydrogen atom in the presence of a homogeneous static electric field $\mathbf{F}=F \mathbf{z}$ is separable in parabolic coordinates [22]. Separability is achieved by writing the wave function in the product form $\psi(\mathbf{r})=[2 \pi \chi v]^{-1 / 2} X(\chi) Y(v) e^{i m \varphi}$ (with $m=0, \pm 1, \pm 2, \ldots$ the magnetic quantum number). Then, the Schrödinger equation splits into two differential equations concerning the functions $X$ and $Y$, respectively, which are (in a.u.)

$$
\begin{aligned}
& {\left[-\frac{1}{2} \frac{d^{2}}{d \chi^{2}}+U_{X, \mathrm{eff}}(\chi)-2 Z_{1}\right] X(\chi)=0,} \\
& U_{X, \mathrm{eff}}(\chi)=\frac{4 m^{2}-1}{8 \chi^{2}}+\frac{F \chi^{4}}{2}-E \chi^{2}, \\
& {\left[-\frac{1}{2} \frac{d^{2}}{d v^{2}}+U_{Y, \mathrm{eff}}(v)-2 Z_{2}\right] Y(v)=0} \\
& U_{Y, \mathrm{eff}}(v)=\frac{4 m^{2}-1}{8 v^{2}}-\frac{F v^{4}}{2}-E v^{2}
\end{aligned}
$$

where the separation constants $Z_{1}$ and $Z_{2}$ are connected through $Z_{1}+Z_{2}=Z$. The problem is solved for given, fixed $E, m$, and $F$ sets.

The form of the effective potential $U_{X \text {,eff forces the bound }}$ electron motion along the $\chi$ coordinate. The small- $\chi$ asymptotic behavior of wave functions $X$ is

$$
\underset{\chi \rightarrow 0}{X(\chi)} \rightarrow A_{X} \chi^{|m|+\frac{1}{2}}\left[1+O\left(\chi^{2}\right)\right]
$$

where the normalization constant $A_{X}$ is positive by definition. Furthermore, the solution of Eq. (4a) ensuring the proper $X \rightarrow 0$ wave function behavior for $\chi \rightarrow \infty$ involves the quantization of $Z_{1}$. The obtained $Z_{1}^{n_{1},|m|}$ (and $Z_{2}^{n_{1},|m|}$ ) set is characterized by the number $n_{1}=0,1,2, \ldots$ of nodes of the corresponding wave functions $X_{n_{1},|m|}$ in the interval $(0, \infty)$. The latter functions are normalized to unity and it turns out that $A_{X}$ decreases slowly with increasing energy and becomes negligible for $Z_{1}(\varepsilon)<0$.

The small- $v$ asymptotic behavior of the wave functions $Y$ is identical to that of Eq. (5), i.e., $\underset{v \rightarrow 0}{Y(v) \rightarrow}$ $A_{Y} v^{|m|+1 / 2}\left[1+O\left(v^{2}\right)\right]$. Similarly to $A_{X}$, the normalization constant $A_{Y}>0$ is negligible when $Z_{2}(\varepsilon)<0\left(Z_{1}>Z\right)$ and stabilizes to a constant value at large $\varepsilon$. Thus, the wave function of each particular $n_{1}$ channel exhibits appreciable amplitude solely within the energy range imposed by the classically allowed $Z \geqslant Z_{1}^{n_{1},|m|} \geqslant 0$ interval. On the other extreme of large $v$ we may write $[22,23]$

$$
\underset{v \rightarrow \infty}{Y} \propto \frac{1}{k^{1 / 2}(v)} \sin [\theta(v)+\varphi],
$$

where $k(v)=\left\{2\left[2 Z_{2}^{n_{1},|m|}-U_{Y, \text { eff }}(v)\right]\right\}^{1 / 2}$ is the wave number function. In Eq. (6),

$$
\theta(v)=\int_{v_{0}}^{v} k\left(v^{\prime}\right) d v^{\prime}
$$

and $\phi$ is a $v_{\mathrm{o}}$-dependent constant phase which carries information for the inner part of $Y$ and particularly $A_{Y}$.

Consider next the photoexcitation of the Stark states $\psi_{n_{1}, m}^{E, F}$ (for $\varepsilon \geqslant-1$ ) out of an initial state $\psi_{i}$. The resulting outgoing flux of ionized electrons is described by the current probability density $J_{v}(\chi, \varphi) \propto$ $i\left[\chi^{2}+v^{2}\right]^{-1 / 2}\left[\psi\left(\partial \psi^{*} / \partial v\right)-\psi^{*}(\partial \psi / \partial v)\right], \quad$ along a paraboloid of constant $v=v_{\text {det }}$. For $v_{\text {det }} \rightarrow \infty$ and for the whole range of values of importance for the electron impact radius $\rho \propto \chi$, this paraboloid practically coincides with the PSD plane at $z_{\text {det }}$ and the expression for the PM image is written as

$$
J_{v_{\mathrm{det}}}(\varphi, \rho) \propto \frac{1}{\chi}\left|\sum_{n_{1}, m} e^{i\left[\theta_{n_{1},|m|}\left(v_{\mathrm{det}}\right)+\phi_{n_{1},|m|}\right]} d_{n_{1}, m} e^{i m \varphi} X_{n_{1},|m|}(\chi)\right|^{2},
$$

where $d_{n_{1}, m}=\left\langle\psi_{n_{1}, m}^{E, F}|\hat{T}| \psi_{i}\right\rangle$ are (single- or multiphoton) dipole transition matrix elements between $\psi_{n_{1}, m}^{E, F}$ and $\psi_{i}$ and $\hat{T}$ stands for a relevant transition operator. Details about the computation of the wave functions $X_{n_{1},|m|}$, the phases $\theta_{n_{1},|m|}\left(v_{\mathrm{det}}\right)+\phi_{n_{1},|m|}$ and the matrix elements are given in Ref. [16].

All the important observables are derived from Eq. (8). The radial distribution $R(\rho)$ is obtained by angularly integrating $J_{v_{\text {det }}}$ over the full $0 \leqslant \varphi \leqslant 2 \pi$ interval. Note that if only $m=0$ final states are excited, $J_{v_{\text {det }}}$ is independent of $\varphi$. Then, the integration is trivial and leads to $R(\rho) \propto J_{v_{\text {det }}}(\rho)$. Moreover, the total ionization cross section is obtained by integrating $J_{v_{\text {det }}}$ over the whole PSD surface. This leads to

$$
\sigma_{\text {tot }} \propto \sum_{n_{1}, m}\left|d_{n_{1}, m}\right|^{2}
$$

As for the glory signal, it is simply given by the value of $J_{v_{\text {det }}}$ at the center of the image $(\chi=0)$. With the help of Eq. (5) this signal is written as

$$
J_{\text {Glory }} \propto\left|\sum_{n_{1}} d_{n_{1}, 0} A_{X, n_{1}, 0} e^{i\left[\theta_{n_{1}, 0}\left(v_{\mathrm{det}}\right)+\phi_{n_{1}, 0}\right]}\right|^{2}
$$

and obviously only $m=0$ waves contribute to it (in agreement with the classical picture where the glory effect appears only for zero projection of the orbital angular momentum on the field axis).

For $-1 \leqslant \varepsilon<0$ the potential $U_{Y \text {,eff }}$ exhibits a barrier and supports both continuum states (where the electron escapes over this barrier) and quasibound ones (where the electron may escape solely via tunneling through the barrier). The latter states are also termed as "resonances" and they are fairly long-lived. They may be further classified according to their localization in space and consequent semiclassical quantization conditions (for details see Refs. [24,25]). On the

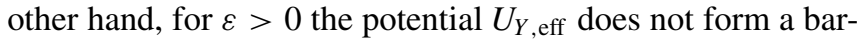
rier and cannot support any quasibound states. Nevertheless, 
$n_{1}$-channel "switching-on" and "switching-off" continues to occur due to the aforementioned behavior of the normalization constants $A_{Y}$ and $A_{X}$, respectively, with energy and this leads to a modulated total ionization cross section. Therefore, a simple approximate way to predict the locations of these resonantlike modulations is by imposing energy quantization along the $\chi$ coordinate for the fixed $Z_{1}=Z$ value $[26,27]$ (channel switching-on). Since these positive energy structures have no counterpart when the static field is absent, they were recently referred to as static-field-induced-states (SFIS) $[24,25]$. Experimentally they were first observed in the positive energy spectra of nonhydrogenic atoms [26] and subsequently in the $\varepsilon>0$ range of the hydrogenic ones [28,29] for $m=0$ final Stark states and static field strengths of several $\mathrm{kV} / \mathrm{cm}$. In either case, the SFIS periodicity is expected to remain the same [30,31]. On the other hand, for $m \neq 0$ and/or weak fields the experimental and theoretical [2,30,3237] results showed quite weak or no modulation contrasts at all. Furthermore, even for $m=0$, the contrast was found to critically depend on the particular initial state and excitation scheme as well [16,38]. An example employing the same field as in Fig. 3(a) is given in the $\sigma_{\text {tot }}$ calculation of Fig. 3(b), corresponding to two-photon excitation of $m=0$ final Stark states out of the hydrogenic ground state. The spectrum shows both quasibound state and SFIS resonances superimposed over an appreciable background. The field strength is weak (albeit compatible with present day PM studies [16]) and this results in positive energy structures with a quite low modulation contrast.

Remarkably, quasi-bound state or SFIS imprints of much higher contrast than in $\sigma_{\text {tot }}$ are evident in the quantum mechanically calculated glory spectrum of Fig. 3(c). A first comparison between this glory curve and its classical and semiclassical counterparts of Fig. 3(a) reveals the common presence of local overall maxima around $\varepsilon=-1$ and (mainly) around $\varepsilon=\varepsilon_{\mathrm{dir}}$. Additionally, the periodicity of this signal at positive energy is very close to that of $\sigma_{\text {tot }}$ and apparently the same as the semiclassical glory curve. The quantum beating structures, however, are evidently much more complicated and richer, occasionally comprising almost complete cancellations of the glory signal. These differences should be attributed to the equal weighting among the interfering terms employed in the semiclassical calculation of Eq. (3), as opposed to the unequal weighting implied by Eq. (10). In fact, the dependence of the glory signal on the transition matrix elements $d_{n_{1}, 0}$ makes its detailed structure strongly dependent on the initial state and the excitation scheme.

Let us conclude this first comparison between calculated results by a presentation of the secondary bow signal $J_{\text {sbow }}$. This signal should be strictly defined as $J_{\text {sbow }} \equiv J_{v_{\text {det }}}\left(\rho_{I I}\right)$ and, according to recent suggestions [13,16,38], $\rho_{I I}$ may be associated with the outermost inflection point $\rho_{\text {ip }}$ of the radial distribution $R(\rho)$. In turn, the radius $\rho_{\text {ip }}$ follows in general closely the simple analytic expression of $\rho_{\max }^{\mathrm{cl}}[3-5]$, but additionally exhibits some localized abrupt magnitude variations. For $\varepsilon<0$ these variations are due to either the transformation of $n_{1}$ channels to continua or the presence of resonances [38], while for $\varepsilon>0$ they are due to the presence of SFIS [16]. For the purpose of obtaining the bow signal over a smoothly energy-varying and atom-independent radius we adopt throughout in the present work the approximation $\rho_{I I} \approx$ $\rho_{\max }^{\mathrm{cl}}$, which is very good for $\varepsilon<0$ and excellent for $\varepsilon \geqslant 0$ [4,5]. The outermost inflection point of $R(\rho)$ at $\varepsilon=0$ is then just employed for scaling purposes and this $\rho_{\max }^{\mathrm{cl}}(\varepsilon=0)=$ $\rho_{\text {ip }}(\varepsilon=0)$ scaling is followed for either the computed or the experimental data. Tests involving radii differing slightly from the scaled $\rho_{\max }^{\mathrm{cl}}$ (say $\rho=\rho_{\max }^{\mathrm{cl}} \pm \Delta \rho$, with $\Delta \rho / \rho_{\max }^{\mathrm{cl}} \approx$ $5 \%$ at $\varepsilon=0$ ) resulted in practically identical secondary bow curves (apart from small magnitude differences). Therefore, for fairly small $\Delta \rho$, the choice of the secondary bow radius is not critical (as long as it behaves smoothly with energy) and our approximation is justified. The so obtained $J_{\text {sbow }}$ curve is given in Fig. 3(d). Clearly this signal and the $J_{\text {Glory }}$ spectrum of Fig. 3(c) appear to contain equivalent and perhaps even complementary information on quantum interferences. For example, within the reduced energy range $-0.2<\varepsilon<$ +0.2 intensity maxima of one signal coincide with the other's minima, thus reproducing qualitatively the behavior noticed in the experimental data of Fig. 1(a). Nevertheless, the glory signal is much stronger than the corresponding secondary bow one [compare the $y$ axes of Figs. 3(c) and 3(d)].

\section{EXPERIMENTAL SETUP AND PROCEDURE}

Details about the experiment were previously provided in Refs. [16,38] and we will only give a brief description here. Magnesium vapor is produced in an oven mounted at the top of the laser-atom interaction chamber held at a background pressure of $\approx 7 \times 10^{-7}$ mbar. An atomic beam is formed which enters the interaction chamber. Magnesium atoms are excited from their $3 s^{2} S_{0}$ ground state to the vicinity of the ionization threshold (IP(Mg) $=61671.05 \mathrm{~cm}^{-1}$ [39]) by two-photon nonresonant excitation in the $305-335 \mathrm{~nm}$ UV range. This radiation is produced by frequency doubling the 610-670 nm output light of a Nd:YAG pumped dye laser using a KDP crystal. The laser operates at a repetition rate of $10 \mathrm{~Hz}$. The fundamental visible radiation pulses have $\sim 5 \mathrm{~ns}$ duration and a linewidth of $\sim 0.2 \mathrm{~cm}^{-1}$. A small part of it is sent to a frequency calibration system providing the fringes of a Fabry-Perot interferometer with a free spectral range of $0.4729(2) \mathrm{cm}^{-1}$ and the optogalvanic spectrum of a $\mathrm{Ne}$ discharge lamp. Judging from the smallest recorded spectral width of the two-UV-photon excited Stark resonances, our overall spectral resolution is about $0.5 \mathrm{~cm}^{-1}$. The linear polarization of the UV radiation is purified and rotated by passing it through a Rochon prism linear polarizer and a double-Fresnel rhomb (acting as a $\lambda / 2$ retarder), respectively. Finally, the UV light is focused in the interaction region through a $\approx 20 \mathrm{~cm}$ focal length lens. The atomic and laser beams are perpendicular to each other and to the static electric field oriented along the axis of the electron spectrometer. The latter is based on the standard three-electrode velocitymap-imaging (VMI) design [40]. The interaction region is positioned midway between the first solid repeller electrode held at voltage $V_{\mathrm{R}}$ and the second hollow extractor one held at $V_{\mathrm{E}}$. This structure is completed by a third grounded hollow electrode and operates as a lens due to the resulting electric field inhomogeneity (VMI $V_{\mathrm{E}} / V_{\mathrm{R}}$ condition $[40,41]$ ). In the vicinity of the limited laser-atom interaction volume, however, 
the static field may be considered as nearly constant, albeit difficult to accurately estimate. Photoelectrons are accelerated by the field towards the end of a field-free drift tube. An electrostatic Einzel lens is placed about halfway through the tube for magnifying the recorded images by a factor up to 20. Photoelectrons are detected by a two-dimensional PSD made of a tandem microchannel plate assembly followed by a phosphor screen. In all experiments we took great care in working at low signal intensity, avoiding as much as possible saturation effects by staying in the linear regime of the MCPs. In addition, working systematically at low signal levels, well below saturation, avoids detector fatigue and prevents the deterioration of its sensitivity in regions where the signal is particularly intense, such as in the center of the image. This is confirmed by the day-by-day reproducibility of the measurements over overlapping energy ranges, as well as by the reproducibility obtained with new channel plates. A CCD camera records the 2D distribution of light spots on the screen. Recorded images are transferred to a PC, where they are accumulated over several thousand laser shots. The entire spectrometer is shielded by a double $\mu$-metal layer, which results in a residual magnetic field $<1 \mu \mathrm{T}$ within its interior.

\section{RESULTS AND DISCUSSION}

\section{A. Experimental observations}

Experimental images from two-photon ionization of $\mathrm{Mg}$ atoms were recorded with the linear laser polarization parallel to the direction of the static electric field. Consequently, due to the $\Delta m=0$ dipole selection rule only $m=0$ final Stark states can be excited, which allow for the emergence of the glory effect. The static field strength was estimated by an array of methods described in Refs. [13,16,38]. Briefly, a rough estimate of $E_{s p}^{\mathrm{cl}}$ and consequently of $F$ was obtained from the lowest energy where an image of quantifiable signal could be recorded. As a second estimate, the energy evolution of the outermost inflection point $\rho_{\text {ip }}$ of the radial distribution $R(\rho)$ was fitted to the expression for $\rho_{\max }^{\mathrm{cl}}[3-5]$ for all the images where the direct contribution was observable $\left(\varepsilon>\varepsilon_{\mathrm{dir}}\right)$, including the positive energy data. Finally, a third estimate was based on the $|m|$ dependence of the classical saddle point energy [42]. Setting the laser polarization perpendicular to the field axis allows for the additional excitation of $|m|=2$ final Stark states. The $|m|=2$ threshold is then estimated by the energy evolution of the angular distribution of these images [38]. All methods converged to $F=680 \pm 10 \mathrm{~V} / \mathrm{cm}$ $\left(E_{s p}^{\mathrm{cl}}=-160 \pm 1 \mathrm{~cm}^{-1}\right)$.

The two-photon excitation energy was scanned by increments of $\Delta E \approx 0.4 \mathrm{~cm}^{-1}\left(\Delta \varepsilon \approx 2 \times 10^{-3}\right)$, covering the entire $-1 \leqslant \varepsilon \leqslant 1$ range. A movie is available in the Supplemental Material displaying the full series of images, a typical sample of which is already given in Fig. 1(a) [43]. The examination of the sequence of images reveals the spatial movement of the interference fringes as a function of energy, along with the modulation of their magnitudes for $\rho<\rho_{I}$ as caused by strong beating effects between the direct and indirect contributions. The glory signal behaves similarly and this can be viewed in the full set of measurements given in Fig. 4(a). More precisely, this plot [as well as the plot of Fig. 1(b)] shows the energy evolution of the glory signal

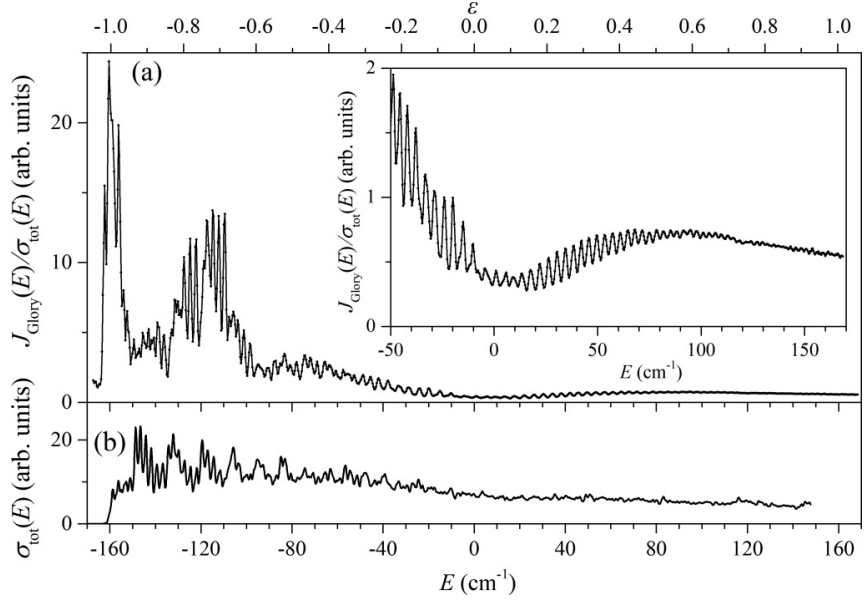

FIG. 4. (a) Energy dependence of the glory signal recorded by employing two-photon near-threshold ionization of ground state $\mathrm{Mg}$ atoms under the presence of a static electric field $F=680 \pm$ $10 \mathrm{~V} / \mathrm{cm}$. The energy scale at the bottom of the figure refers to the zero-field limit and the upper $x$ axis to the reduced energy $\varepsilon$. The glory intensity is computed by integrating the corresponding experimental images over a radius equal to $\approx 2 \%$ of $\rho_{\max }^{\mathrm{cl}}(\varepsilon=0)$. Nevertheless, even the signal at the single central point of each image produces an identical (albeit somewhat noisier) curve. Furthermore, for eliminating any dependence on the variation of the laser pulse energy during the recordings, $y$ axis actually refers to the glory intensity scaled by the total electron signal. The latter is calculated by integrating over the full detector surface and it is proportional to the total cross section $\sigma_{\text {tot }}$. The inset displays a magnified view of the scaled glory signal at positive energies. (b) $\mathrm{The}^{\mathrm{Mg}}{ }^{+}$spectrum, also proportional to $\sigma_{\text {tot }}$ and reproducing the total electron signal. Note that the line strengths of the observed sharp spectral lines near $E_{s p}^{\mathrm{cl}}$ $\left(\approx-160 \mathrm{~cm}^{-1}\right)$ are modulated by envelopes whose locations fall near the predicted SFIS locations [26]. On the contrary, SFIS oscillations near the zero-field threshold and at positive energy are not observed.

scaled by the total electron signal [16], the latter obtained by integrating over the whole surface of the detector. By keeping the laser intensity to an appropriately low level $\left(<10^{10} \mathrm{~W} / \mathrm{cm}^{2}\right)$, this signal was intentionally kept unsaturated in order to be proportional to $\sigma_{\text {tot }}$. In fact, the total electron signal is also completely equivalent to the $\mathrm{Mg}^{+}$ signal shown in Fig. 4(b). The $\mathrm{Mg}^{+}$spectrum was recorded by operating the VMI microscope as a simple time of flight spectrometer with voltages of reversed polarity applied to its interaction region electrodes and otherwise identical conditions.

Due to the small energy step employed for the measurements, the recording of about 800 images lasted several days. As a result, it was found impossible to avoid small gradual drifts of laser pulse energy with time. Hence, the ratio $J_{\text {Glory }} / \sigma_{\text {tot }}$ was employed for avoiding magnitude variations of the glory signal caused by these drifts. As already pointed out earlier [16], however, the scaling may reduce the strength of the manifestation of quasibound states but it cannot affect the details of the interference and beating patterns discussed here. In particular, it is obvious in Fig. 4(b) that positive energy (SFIS) modulations are not observed in $\sigma_{\text {tot }}$. We may anticipate that these modulations are masked by noise due to their 
low contrast. In turn, this low contrast may be the result of the employed excitation scheme in conjunction with the low field strength, since such modulations were observed in $\mathrm{Mg}$ atom only at very high field strengths [44]. Nevertheless, the consequence here is that the shape and periodicity of the $\varepsilon \geqslant 0$ glory oscillations with energy remain unaltered by the scaling operation. On the contrary, a comparison between $J_{\text {Glory }} / \sigma_{\text {tot }}$ and $\sigma_{\text {tot }}$ in the vicinity of $E_{s p}^{\mathrm{cl}}$ shows that, in general, the glory exhibits features that may be attributed to the presence of near-threshold resonances (whose tunneling properties are fairly weak in $\mathrm{Mg}$ atom [38]), but the scaling helps in partially "decorrelating" its magnitude from them. Finally, another consequence of the scaling observed in Fig. 4(a) is the very large value of $J_{\text {Glory }} / \sigma_{\text {tot }}$ near $\varepsilon \approx-1$. This is due to the quite small (quasizero) value of $\sigma_{\text {tot }}$ at this energy range. In fact, the nonscaled glory signal shows indeed a local maximum at threshold, but this maximum is weaker than the local one at $\varepsilon \approx \varepsilon_{\mathrm{dir}} \approx-0.775$. This reversal of magnitudes due to scaling has been already reported in the classical treatment of the glory signal (see Fig. 11 of Ref. [4]).

\section{B. Comparison between experimental and calculated quantities}

Apart from the above-mentioned common feature of $J_{\text {Glory }} / \sigma_{\text {tot }}$ with the scaled classical signals, the data of Fig. 4(a) show magnitude oscillations and beating structures just like the semiclassical curve of Fig. 3(a) does. They exhibit, however, an even closer overall qualitative resemblance with the hydrogenic glory signal of Fig. 3(c) calculated quantum mechanically at the same field strength. This resemblance calls for a closer comparison with the scaled version of the latter calculation. Experimental and calculated scaled glory signals are given in Fig. 5(a). For the purpose of unveiling the global, atom-independent features of these signals, the comparison is restricted to the vicinity of the zerofield limit and the positive energy range (the lower energy spectral structures due to quasibound states are expected to be more strongly atom specific). As the figure shows, at negative energy the two scaled glory signals are somewhat different, the theoretical one being more complicated since its oscillation appears to be double peaked. Their agreement gets much better at higher energy, where they both show single-peaked oscillations, which are of the same periodicity but slightly out of phase. Their periodicity is energy dependent and the distance between successive maxima decreases from $\sim 5 \mathrm{~cm}^{-1}$ at $E \approx 0$ to about $3 \mathrm{~cm}^{-1}$ at $E \approx 100 \mathrm{~cm}^{-1}$ $(\varepsilon \approx+0.625)$. Furthermore, a complete cancellation of the oscillations in the theoretical curve occurs at $E \approx 20 \mathrm{~cm}^{-1}$ $(\varepsilon \approx+0.125)$, while the experimental one exhibits a similar cancellation at $E \approx 5 \mathrm{~cm}^{-1}(\varepsilon \approx+0.031)$. The theoretical curve exhibits several additional partial cancellations [see the inset of Figs. 3(c) and 5(a) around $E \approx 75 \mathrm{~cm}^{-1}$, i.e., around $\varepsilon \approx+0.469$ ). Tests with calculated hydrogenic signals show that the number and location of cancellations are initial state and excitation-scheme specific. Clearly they should depend on the target atom as well.

Let us now briefly discuss the similarities and differences between the experimental and calculated $J_{\text {sbow }} / \sigma_{\text {tot }}$ curves given in Fig. 5(b) (external ring, secondary bow signal). At a first glance, these curves appear to carry similar information

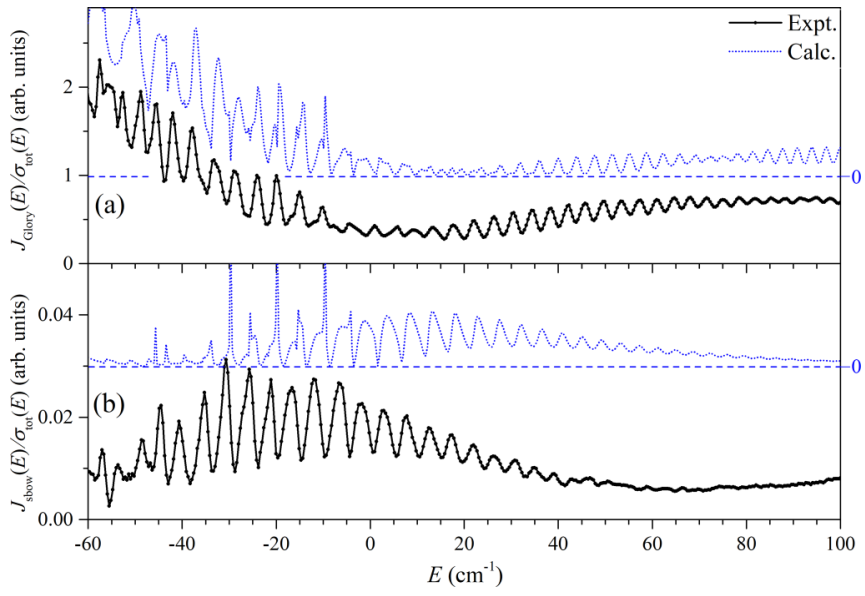

FIG. 5. (a) Comparison between the experimental scaled glory signal (two-photon ionization of $\mathrm{Mg}$ ground state, solid lines and points) and the scaled quantum mechanical one (two-photon excitation out of the hydrogenic ground state, short-dashed lines), within a limited energy range that includes the zero-field limit. The static electric field is $F=680 \pm 10 \mathrm{~V} / \mathrm{cm}$ and the linear light polarization is parallel to the field axis ( $m=0$ final Stark states). For better visibility an offset has been applied to the theoretical curve and its zero is given in the right $y$ axis. (b) The corresponding scaled secondary bow signals. The latter are defined as $J_{\text {sbow }} / \sigma_{\text {tot }} \equiv J_{v_{\text {det }}}\left(\rho_{I I}\right) / \sigma_{\text {tot }}$, where the approximation $\rho_{I I} \approx \rho_{\max }^{\mathrm{cl}}$ is adopted and where the maximum classical radius is scaled to the outermost inflection point of the radial distribution $R(\rho)$ at $E=0$. Note that the intensity $y$-axis units are arbitrary but common to (a) and (b) and that in both graphs experimental and theoretical curves exhibit the same periodicity but the oscillations are phase shifted and beating minima and maxima generally occur at different energy locations.

with the glory signals. Particularly, apart from the small differences in their detailed structure (attributed to the different target atoms), they exhibit the same periodicity among themselves. Moreover, their periodicity is quite close to that of the $J_{\mathrm{Glory}} / \sigma_{\mathrm{tot}}$ curves. However, bow and glory signals are found to be completely out of phase by $\pi$ at negative energy and this has been already pointed out in the experimental images of Fig. 1(a). They get in phase, however, at positive energy. Additionally, the magnitude of the oscillations of the secondary bow signal is maximized near the location of the beating minimum of the glory curve. This can be also noticed in the calculation of Figs. 3(d) and 5(b). In fact, the only important difference between experimental and theoretical data lies at the somewhat different aforementioned locations where the effect occurs. Hence, a first quite general finding is already emerging at this point. Whether it is the scaled glory signal, or the scaled secondary bow signal, the agreement between experiment and theory in the positive energy range is extremely good. This fact underlines the general character of these oscillations insofar as the quantum hydrogenic calculation does not take into account the effects stemming from the presence of $\mathrm{Mg}^{+}$residual ionic core of non-negligible size.

As suggested in Ref. [6], the interference phenomena exhibited by the photoelectron momentum distribution $R(\varepsilon, \rho)$ may, under certain conditions, provide information on the ionization time delays between classes of electron trajectories. 
One may then anticipate that, specifically for the glory signal $R(\varepsilon, \rho=0)$, this information should concern the arrival time difference between those classical electron trajectories that end up at the detector center. In the present work we attempt to bring out such kind of information by applying the so-called "short time Fourier transform" (STFT) to the scaled glory signals. STFT is customarily employed in order to determine the (possibly varying) frequency and phase content of local sections of a signal changing with time [45]. A selected window function is slid along the time axis and the Fourier transform is applied solely within the interval covered by the window. In practice, for reducing artifacts at the boundaries, there is a certain degree of overlap between successive window locations. The outcome of such a procedure is a two-dimensional representation of the frequency content as a function of time. Note that in our case the two conjugated variables are still time and frequency (energy), but they are interchanged and the necessity to use STFT stems from the energy-varying periodicity of the glory oscillation. After some experimentation, a fair compromise between frequency and time resolution (at least for $E \geqslant 0$ ) was found to be an energy window length of $\approx 21 \mathrm{~cm}^{-1}$ that corresponds to a time uncertainty of $\approx 1.6 \mathrm{ps}$. It was also found advantageous to choose a Blackman window function [46]. A smooth STFT distribution is obtained by setting the overlap between adjacent energy windows to be the largest possible, i.e., comparable to the window width. The so-obtained two-dimensional STFT representations for the experimental scaled glory signal of $\mathrm{Mg}$ and the computed hydrogenic one [scaled version of that plotted in Fig. 3(c)] are given in Figs. 6(a) and 6(b), respectively. As a first remark, it is obvious that a meaningful comparison is quite difficult to make at negative energies, where the representations are dominated by atom- and excitation scheme-specific features. The situation is not improved even with a smaller energy window length that is more compatible with the negative energy glory structures. On the contrary, the two graphs reveal a common time-frequency branch at $t \approx 0$ and a quite similar structure at positive energy. The $t \approx 0$ horizontal branch corresponds to the "DC" Fourier component at each energy window location. It reflects the "envelope" of the glory spectrum, that is, its average over the window length as a function of energy. As for the $t>0$ positive energy range, the representations consist of several other branches evolving quasilinearly with $E$ (this way confirming the aforementioned varying periodicity of the glory signal). The slopes of the two branches observed in the experimental data [Fig. 6(a)], as well as their values at $E \approx 0(\approx 7$ and $\approx 14$ ps, respectively $)$ are practically identical to those observed in the theoretical representation of Fig. 6(b). The latter shows one more branch for $t \leqslant 30 \mathrm{ps}$. In general, the highest the slope the faintest the branch, thus necessitating the employment of a logarithmic color scale covering five orders of magnitude. It is also interesting to note that the linear branches exhibit beating modulations of their magnitude. This is particularly evident in the theoretical data, and the locations of the local minima differ from one branch to another. Interestingly, the minima concerning the first, lowest slope, quasilinear branch coincide with the beating local minima of the glory signal itself [see, for example, Fig. 3(c) and especially, compare Figs. 5(a) and 6(b) around $E=20$ and $80 \mathrm{~cm}^{-1}$ ]. This beating structure
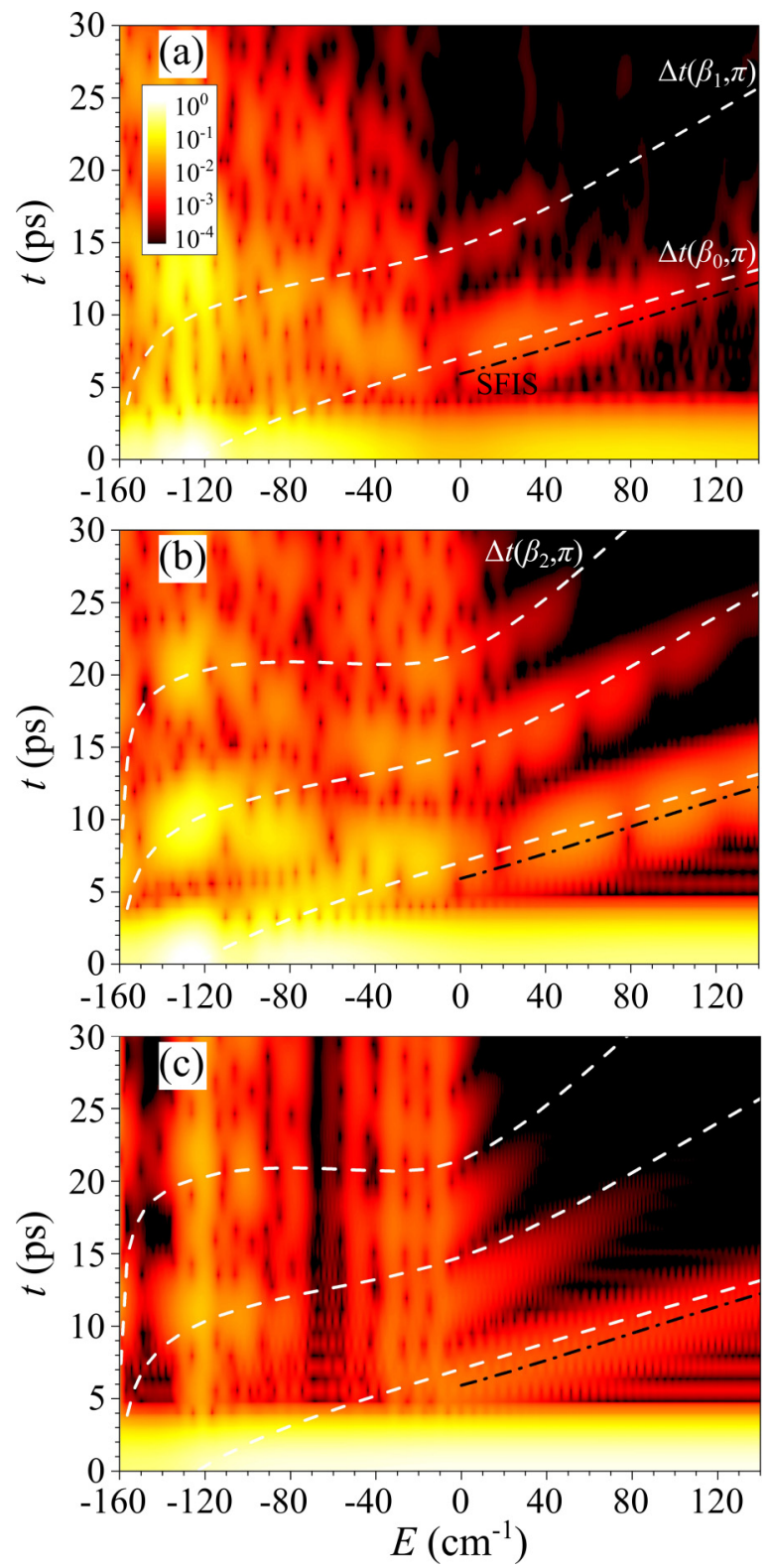

FIG. 6. (a) Short time Fourier transform (STFT) applied to the $\mathrm{Mg}$ scaled glory signal given in Fig. 4(a). The employed energy window is $\approx 21 \mathrm{~cm}^{-1}$ and results to a time resolution of $\approx 1.6 \mathrm{ps}$. Drawn with white dashed lines are the classically computed [6] differences $\Delta t\left(\beta_{k}, \pi\right)$ between the arrival times on the detector for the electron trajectories corresponding to launch angles $\beta=\pi$ and $\beta=\beta_{k}$, for $k=0,1$. The black dashed-dotted line corresponds to the prediction of Eqs. (12), which is based on SFIS periodicity. (b) Same as in (a) but for the scaled version of the glory signal of Fig. 3(c), corresponding to two-photon excitation of hydrogen out of its ground state ( $m=0$ final Stark states). In addition to the time differences $\Delta t\left(\beta_{0}, \pi\right)$ and $\Delta t\left(\beta_{1}, \pi\right)$, the classically computed difference $\Delta t\left(\beta_{2}, \pi\right)$ is also plotted as a function of energy. (c) STFT applied to the calculated hydrogenic total ionization cross section of Fig. 3(b), along with the differences $\Delta t\left(\beta_{k}, \pi\right), k=0-2$ and SFIS-based prediction of Eqs. (12), as in (a) and (b).

may be the result of an interference effect involving multiple closely spaced "frequencies", a hypothesis which is consistent with the width of each branch. 
Let us now examine more quantitatively the connection between time delays and the glory signals. To that purpose we superimpose on the representations of Fig. 6 with white dashed lines the classically calculated [6] time differences $\Delta t\left(\beta_{k}, \pi\right) \equiv T\left(\beta_{k}\right)-T(\pi)$, where $T(\pi)$ and $T\left(\beta_{k}\right)$ are the flight durations of an electron launched from the origin with angles $\beta=\pi$ and $\beta=\beta_{k}$, respectively, which, as discussed in the theoretical section and as can be visualized in Fig. 2, all lead to a zero impact radius on the detector. Note that the time difference $\Delta t\left(\beta_{k}, \pi\right)$ does not depend on the source-todetector distance as long as this distance largely exceeds the atomic dimensions (typically the distance between the saddle point and the origin). Here it is evaluated at a "macroscopic" distance of $1 \mathrm{~mm}$. The time difference contains no adjustable parameters and leads to an obviously excellent agreement with either the experimental data (for $k=0,1$ ) or the quantum mechanically computed hydrogenic ones $(k=0,1$, and 2$)$. The quasilinearity discussed earlier for the positive energy range is here more evident. To a good approximation this part of the curves $(0<\varepsilon<1)$ can be described by straight lines of the form $\Delta t_{k}=\alpha_{k}(\varepsilon+1)$, where the slopes $\alpha_{k}$ appear to be proportional to $k+1$. Note, however, that at higher energy $(\varepsilon>1)$ the curves become similar to the photodetachment case and $\Delta t_{k}$ 's depart largely from a linear evolution. Detailed classical calculations of electron times of flight as well as a general discussion on the behavior of the various relevant classical delays as a function of energy will be discussed thoroughly in a forthcoming paper.

It is finally instructive to compare the periodicities of the glory signal and the SFIS structures occurring in $\sigma_{\text {tot. }}$. We can find an expression for the SFIS periodicity by means of the aforementioned semiclassical energy quantization condition along the $\chi$ coordinate with $Z_{1}=Z$ and the suggestions of Ref. [47]. Thus, we start by defining the SFIS phase as

$$
S_{\mathrm{SFIS}} \equiv 2 \int_{0}^{\chi+}\left(-F \chi^{4}+2 E \chi^{2}+4 Z\right)^{1 / 2} d \chi-\pi
$$

with the outer turning point given by

$$
\chi_{+}=2^{1 / 2}\left(\frac{Z}{F}\right)^{1 / 4}\left[\varepsilon+\left(\varepsilon^{2}+1\right)^{1 / 2}\right]^{1 / 2} .
$$

The hydrogenic SFIS energy levels $E_{\mathrm{SFIS}}^{n_{1}}$ are obtained by solving the equation $S_{\mathrm{SFIS}}^{n_{1}}=2 n_{1} \pi[26-28,36]$. Now the SFIS periodicity, expressed in terms of a time difference $\delta t_{\text {SFIS }}$, is given by

$$
\begin{aligned}
\delta t_{\mathrm{SFIS}} \equiv & \frac{\partial S_{\mathrm{SFIS}}}{\partial E}=\frac{2^{3 / 2} Z^{1 / 4}}{F^{3 / 4}}\left[\left(1+\varepsilon^{2}\right)^{1 / 2}-\varepsilon\right]^{1 / 2} \\
& \times[\mathrm{E}(\gamma)-\mathrm{K}(\gamma)],
\end{aligned}
$$

where $\mathrm{K}(\ldots)$ and $\mathrm{E}(\ldots)$ are the complete elliptic integrals of the first and second kind, respectively [48], and

$$
\gamma=\frac{\varepsilon+\left(1+\varepsilon^{2}\right)^{1 / 2}}{\varepsilon-\left(1+\varepsilon^{2}\right)^{1 / 2}}
$$

Equation (12a) is also superimposed in the STFT representations of Fig. 6 . Obviously $\delta t_{\text {SFIS }}$ is very close to the periodicity $\Delta t\left(\beta_{0}, \pi\right)$. Nevertheless, it is somewhat smaller and distinctly different from it and describes much less satisfactorily the corresponding linear glory branch of either
Fig. 6(a) or 6(b). On the contrary, and as expected, it fits better than $\Delta t\left(\beta_{0}, \pi\right)$ the first linear branch of the STFT representation of the calculated hydrogenic total cross section $\sigma_{\text {tot }}$ given in Fig. 6(c). The positive energy structure of this representation is similar to that of the glory signal, showing a substantial number of linear branches. In an attempt to interpret the branch described by the time interval $\delta t_{\text {SFIS }}$ of Eq. (12a), let us recall that SFIS states were associated with a bouncing motion of the electron in a resonator formed at $z>0$ by a combination of the atomic potential and the static field [24]. Then, this branch may reflect the round trip time within the cavity, which is intrinsically, though slightly, different from $\Delta t\left(\beta_{0}, \pi\right)$. As for the other, higher slope, branches in Fig. 6(c), they exhibit large discrepancies with the $\Delta t\left(\beta_{k}, \pi\right)$ curves for $k>0$ and their origin and interpretation is not clear yet. They may also, however, approximately be described by straight lines of the form $b_{k}(\varepsilon+1)$ with the slopes $b_{k}$ being proportional to $k+1$. Then it may be anticipated that these branches are connected to multiple round trips.

\section{Synthesis and discussion}

As discussed above, the observations at negative energy are rather complicated to explain globally with simple arguments that allow the derivation of general trends. Therefore, in the following we will focus exclusively on the $\varepsilon \geqslant 0$ energy range, which is of broader interest from this point of view.

Let us first address the question of the (even small) difference between the "carrier frequencies" exhibited by the glory signal and by the total photoionization cross section. A first qualitative answer has been outlined in Sec. IV B. More quantitatively, the origin of this difference lies in the different forms of Eqs. (9) and (10). First, the total cross section as given by Eq. (9) is an incoherent sum depending only on the squared moduli of the matrix elements $d_{n_{1}, 0}$, while the glory signal Eq. (10) is a coherent one, depending on the $d_{n_{1}, 0} \mathrm{~s}$, the phase factors $\exp \left[\theta_{n_{1}, 0}\left(v_{\mathrm{det}}\right)+\varphi_{n_{1}, 0}\right]$ and the normalization constants $A_{X}$. Second, the matrix elements are proportional to both normalization constants $A_{X}$ and $A_{Y}$, while the phases $\theta_{n_{1}, 0}\left(v_{\text {det }}\right)+\phi_{n_{1}, 0}$ carry information connected solely to $A_{Y}$ (through the matching procedure between the inner and outer parts of the wave function $Y(v)$ as described, for example, in Refs. [16,37]). Hence, both the glory signal and the total cross section exhibit the same dependence on $A_{X}$, but the dependence of $J_{\text {Glory }}$ on $A_{Y}$ is much more ( $\sim$ twice) important than that of $\sigma_{\text {tot }} . A_{X}$ is slowly varying with the energy and its role is small. It just smoothly switches-off a given $n_{1}$ channel $\left(Z_{1} \sim 0\right)$. Indeed, by performing several tests we verified that even if this factor is removed from Eq. (10) the STFT representations remain practically unchanged. On the contrary, for $\varepsilon \geqslant 0$ the normalization constant $A_{Y}$ quite abruptly switches-on each channel when $Z_{1} \sim Z$ and it is the principal factor responsible for the produced structures in either $J_{\text {Glory }}$ or $\sigma_{\text {tot }}$. This explains the fair reproduction of the glory oscillations by the semiclassical theory [see Fig. 3(a)], despite the fact that Eq. (3) was employed with equal weights for each wave and does not depend on the $d_{n_{1}, 0}$. Additionally, the aforementioned more complicated dependence of $J_{\text {Glory }}$ on $A_{Y}$ explains the more complicated quantum mechanical glory curve [see Fig. 3(c)]. Note, however, that semiclassical 
computations predict that the difference between the "carrier frequencies" of $J_{\text {Glory }}$ and $\sigma_{\text {tot }}$ strongly decreases at the high energy $(\varepsilon \gg 1)$ and high field strength limit. Consequently, it is expected that at these limits the two oscillations would appear to be almost identical. This is consistent with the findings of recent studies [25] dealing with extreme field strengths, where the oscillations of the transverse momentum distributions and those of SFIS are found to be practically always in phase.

Consider finally the multielectron case where Eqs. (8)-(10) remain unchanged in form and only the $d_{n_{1}, 0}$ s differ with respect to the hydrogenic ones. As it was already pointed out, for $\varepsilon \geqslant 0$ the SFIS oscillations have the same periodicity in either hydrogen or nonhydrogenic atoms. The oscillations of the latter, however, may be identical, slightly shifted [31] or inverted [30] with respect to those of the former, depending on the quantum defects of the target atom. As for the glory signal, the hydrogenic phase factors $\exp \left[\theta_{n_{1}, 0}\left(v_{\text {det }}\right)+\phi_{n_{1}, 0}\right]$ ensure that the hydrogenic frequency components would be present here as well. Since, however, generally the multielectron atom matrix elements become complex [30,49], some modifications with respect to hydrogen are to be expected, as their phases enter in the coherent sum of $J_{\text {Glory }}$ (while this is not important when the matrix element moduli are employed, as in $\sigma_{\text {tot }}$ ). Consequently, the glory signal may exhibit a number of targetatom specific features.

Let us now focus solely on the glory signal and its analysis from a classical point of view. For $\beta \approx \pi$ we have approximately $\rho(\beta) \propto \sin (\beta)$ (as in photodetachment [4]) so the term $\sin (\beta) / \rho(\beta)$ in Eq. (2) is not singular and does not contribute to the glory signal. In the vicinity of the other angles $\beta_{k}$ leading to zero radius we may, to a first approximation, write $\rho(\beta) \propto\left|\sin \left(\beta-\beta_{k}\right)\right|$. Then the term $\sin (\beta) / \rho(\beta)$ is singular and, in principle, all these angles may contribute to the glory signal as long as they differ appreciably from zero. As Fig. 2 shows, however, for $\varepsilon \geqslant 0$ it is only $\beta_{0}$ that has appreciable amplitude. Additionally, for $k>0$ the above approximation seriously underestimates the derivatives $d \rho /\left.d \beta\right|_{\beta \approx \beta_{k}}$ which become very large. Hence, the corresponding $|d \rho / d \beta|^{-1}$ terms in Eq. (2) become very small, diminishing even more these contributions. Consequently, at positive energy $\beta_{0}$ is practically the only one responsible for the glory effect. Nevertheless, this $\beta_{0}$ contribution gives only the main "continuous" component of the glory. If only the electrons ejected at an angle in the vicinity of $\beta_{0}$ contributed to the glory, it would not present any oscillation as a function of energy. Therefore, there must necessarily be interference with other families of trajectories for the oscillations to be observed. In order to determine those trajectories that contribute significantly to the glory modulation we have calculated the individual contributions at the center of the image for $\beta \approx \pi, \beta_{0}$, and $\beta_{1}$ by assuming an isotropic initial angular distribution. Figure 7 shows these partial contributions. They were evaluated by simply calculating the solid angle around the respective launch angle that corresponds to trajectories falling on the detection plane at a radius $\rho<0.1 \rho_{\max }^{\mathrm{cl}}(\varepsilon=0)$, i.e., smaller than $10 \%$ of the maximum classical radius at $E=0$ (the relative contributions at various angles may vary with this fraction, but $10 \%$ was estimated to be a good compromise). Clearly the $\beta_{0}$ contribution is by far the dominant one. Addi-

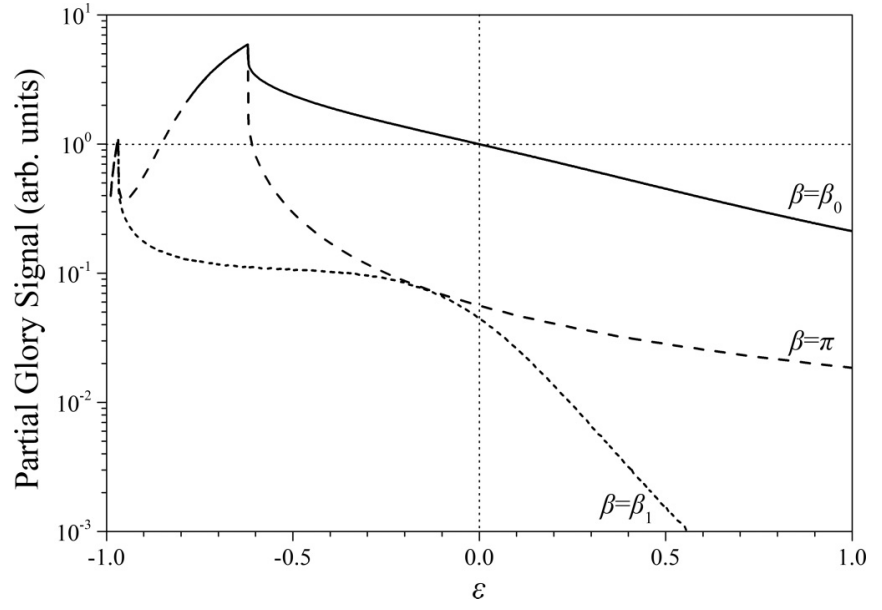

FIG. 7. Individual contributions to the signal at the center of the image for the launch angles $\beta=\pi$ (dashed line), $\beta_{0}$ (solid line), and $\beta_{1}$ (short-dashed line), under the assumption of an isotropic initial angular distribution. Each contribution is obtained by calculating the solid angle around the respective launch angle that corresponds to trajectories falling on the detection plane at a radius $\rho<0.1 \rho_{\max }^{\mathrm{cl}}(\varepsilon=0)$. All contributions are scaled to the partial signal of $\beta_{0}$ at $\varepsilon=0$. Consequently this signal equals unity at the zero-field threshold, as indicated by the dotted-line cross.

tionally, at $\varepsilon \sim 0$ the $\pi$ and $\beta_{1}$ contributions are comparable, while for $\varepsilon>0.5$ even the $\beta_{1}$ contribution is negligible (and the same holds, even more sharply, for $\beta_{k}, k>1$ ). Hence, at $\varepsilon \sim 0$ the oscillations are formed essentially by three waves and this explains the more complicated interference patterns in this region, while for $\varepsilon>0.5$ there are practically just two interfering waves (for $\beta \approx \pi$ and $\beta_{0}$ ). This explains the observation that the $\Delta t\left(\beta_{0}, \pi\right)$ branch in Fig. 6(b) is the strongest one. Nevertheless, the beating effects in this branch appear to arise from an intermodulation among the various classes of trajectories and this is a more subtle effect that can hardly be explained other than by numerical simulation.

It is important to note that the observed oscillations at the center of the image, and the glory effect itself, are two distinct phenomena of quite different origin. For example, in photodetachment where the glory effect is completely absent, there are magnitude oscillations at the center of the images stemming from the interference between the waves corresponding strictly to $\beta=\pi$ and $\beta=0$ trajectories [50]. In our case, at sufficiently high energy, we observe the interference between the waves corresponding to the $\beta=\pi$ and $\beta=\beta_{0}$ trajectories, while the strong intensity of the glory effect arises solely from the $\beta=\beta_{0}$ trajectory. Thus, the emergence of the glory "intensifies" and makes easier the visualization of the interference effect stemming from various trajectories. Consequently, glory oscillations in photoionization are more visible simply because of the high intensity of the glory, whereas the oscillations in the center of the image in photodetachment are hardly visible because of the low signal intensity. Interestingly, in photoionization, the oscillations would persist even at very high positive energy, until the glory effect and its intensifying action would disappear when $\beta_{0}$ becomes practically zero. There, however, it is rather unlikely that the 
observation of these oscillations would be possible, at least at weak fields where the contrast is low.

Finally, it is worth pointing out that the aforementioned quasilinear branch behavior $\Delta t_{k}=\alpha_{k}(\varepsilon+1)$ and particularly the fact that the slopes $\alpha_{k}$ appear to be proportional to $k+1$ corresponds to approximately equal time differences $\Delta t\left(\beta_{0}, \pi\right), \Delta t\left(\beta_{0}, \beta_{1}\right), \Delta t\left(\beta_{1}, \beta_{2}\right)$, etc. It is therefore difficult to decide which trajectory dominates in the construction of the glory oscillations solely on the basis of these time differences. The data of Fig. 7 are necessary to demonstrate that the oscillation is indeed produced by the $\left(\beta_{0}, \pi\right)$ pair.

\section{CONCLUDING REMARKS}

We have presented experimental results on the glory signal at the center of photoionization microscopy images, the latter recorded via near-threshold two-photon ionization of ground state magnesium atoms. The glory signal is found to exhibit strong quasiperiodic oscillations and beating patterns as a function of excitation energy. These data have been analyzed and interpreted by employing classical, semiclassical, and quantum mechanical descriptions of the hydrogenic Stark effect. Attention has been focused on the positive energy range where the observed structures are much less atom and excitation scheme dependent. The quantum mechanical description has first revealed the origin, the similarities, and the differences between the positive energy glory oscillation and the concomitant oscillation of the total photoionization cross section. Subsequently, we have demonstrated the excellent agreement between the experimental $(\mathrm{Mg})$ and calculated $(\mathrm{H})$ glory signal's general behavior and periodicity. Finally, the "short time" Fourier transform of this signal has allowed for the visualization of the close correspondence between spectral features and time delays. The latter refer to classically calculated electron time of flight differences among the various trajectories leading the electron to the center of the image.

The successful employment of a hydrogenic model for the description of the glory signal recorded in a complex atom reveals the global character of this signal; at least as far as the positive energy range is concerned. This "universality," however, needs to be firmly established by performing similar measurements on other atomic systems, a priori heavier than magnesium. These studies should be carried out under perfectly comparable conditions in order to distinguish the truly universal from the atom-specific effects. The latter could be also examined, of course, by means of appropriate Stark theoretical frameworks involving parameters [23,49] and/or atomic potentials [24] characterizing nonhydrogenic atoms. In addition, one could envision other interesting spectral domain extensions of the present work, such as the introduction of an experimentally controllable phase to the glory signal, by applying phase sensitive coherent control techniques to photoionization microscopy [51]. This is expected to allow for higher sensitivity and partial access to the glory signal's phase.

The present study underlines the power of the energy domain analysis of the continuum Stark effect in terms of a classical mechanics approach. Yet, a time domain experiment would evidently constitute a more direct approach for extracting this kind of information without the necessity of any intermediate Fourier transform step. This could perhaps be achieved by devising variants of the beautiful earlier time domain experiments offering resolution at the picosecond scale [52], where the interest will now be centered at the glory signal instead of the total one. On the other hand, such experiments are characterized by an increased difficulty and are, of course, intrinsically limited by Heisenberg's principle.

Let us finally note that our observations on the close connection between spectral features and time delays mentioned above constitute an enlightening illustration of the correspondence principle relating the period of classical motion with the energy differences between successive bound quantum levels. In the present case this correspondence is generalized to the nonperiodic classical motion, while the energy (quasi-) quantization occurs in the continuum. The aforementioned electron time of flight differences are inversely proportional to the energy differences between successive maxima of the glory oscillations.

The above remarks may lead one to envision an existing analogy with Eisenbud-Wigner-Smith (EWS) time delays [53]. This semiclassical concept connects the scattering phase acquired by a wave packet when scattered by a potential with the additional time it takes the particle to reach a given position in space due to the presence of this potential. It may be shown that this additional time corresponds to the derivative of the scattering phase with respect to the particle's energy. The concept can be applied to the half-collision process of photoionization, but the time variation being extremely small, it could be measured only recently thanks to emerging attosecond technology [54,55]. In attosecond photoemission experiments EWS delays are extracted from a measurement of the scattering phase, which requires a phase reference. This is done by simultaneously measuring the phase for two different electronic states of either the same system or of two different systems. Nevertheless, there is a nontrivial correspondence between the measured phase and the scattering phase, while, overall, the time domain interpretation of photoionization experiments combines several notions. For instance, the lifetime of a resonance embedded in the continuum is linked to its spectral width and it may well lie within the fs-ps domain. On the other hand, the EWS delay deduced by attosecond experiments for the same resonance lies in the range of few-fs or sub-fs [56], revealing a different notion. In the case of PM and for a flat continuum the launched electronic wave packet acquires a phase when it scatters in the Coulomb-Stark potential. Semiclassically speaking this corresponds to the phase acquired along an ensemble of classical trajectories. The variation of this phase with energy corresponds to the EWS delay. In a structured continuum, however, the correspondence with the classical trajectories would be certainly more delicate. Overall the analysis of PM experiments in terms of scattering phases and EWS delays is clearly an issue of primary interest that is worth addressing in the near feature.

\section{ACKNOWLEDGMENTS}

The experiment was performed at the Central Laser Facility of the University of Ioannina. Technical assistance from P. Triantafillou and E. Dimitriadis is gratefully acknowledged. The authors also acknowledge the support of this work by the project "ELI - LASERLAB Europe Synergy, HiPER 
\& IPERION-CH.gr" (MIS 5002735) which is implemented under the Action "Reinforcement of the Research and Innovation Infrastructure," funded by the Operational Program "Competitiveness, Entrepreneurship and Innovation" (NSRF 2014-2020) and co-financed by Greece and the European Union (European Regional Development Fund).

\section{APPENDIX: PARAMETER $Q$ FOR $\left|z_{\text {det }}\right|, v_{\text {det }} \rightarrow \infty$}

The classical Coulomb-Stark problem was mostly treated in the $\left(\xi=\chi^{2}, \eta=v^{2}, \varphi\right)$ parabolic coordinate system $[3,27,57]$ while the employment of $(\chi, v, \varphi)$ parabolic coordinates used in the present work is rather limited [19,31] (apart from their extensive and relatively recent use in celestial mechanics [18]). The final expressions are different but the computed trajectories and parameter $Q$ are, or course, the same. Let us restrict ourselves to the planar electron motion and recall that separation in parabolic coordinates requires the introduction of the reduced time variable $\tau$, defined by $d \tau=d t /(2 r)[2,3,18,27,57]$. It turns out that the $\chi(\tau)$ motion is bound and periodic with half period $\tau=\mathrm{T}_{\chi}$ (note that this corresponds to a full period along the coordinate $\xi$ ). Along the $v$ coordinate the reduced time required for the electron to reach the detector (at $v=v_{\text {det }}$ ) is $\tau=\mathrm{T}_{v}$. Therefore, the number $Q$ of half- $\chi$ oscillations performed until the electron reaches the detector can be defined as

$$
Q \equiv \frac{\mathrm{T}_{v}}{\mathrm{~T}_{\chi}} .
$$

If $v_{\text {det }}$ (and consequently $\left|z_{\text {det }}\right|$ ) is finite $Q$ needs to be computed numerically. For $\left|z_{\text {det }}\right|, v_{\text {det }} \rightarrow \infty Q$ can be expressed analytically [27]. For the convenience of the reader we provide this analytic expression here in a rather simpler form, solely in terms of the reduced energy $\varepsilon \geqslant-1$ and the ejection angle $0 \leqslant \beta \leqslant \pi$. It is given by

$$
Q(\varepsilon, \beta)=\frac{\left[\varepsilon^{2}+\cos ^{2}(\beta / 2)\right]^{1 / 4}}{[2 \sin (\beta / 2)]^{1 / 2}} \frac{\mathrm{K}\left(m_{v}\right)}{\mathrm{K}\left(m_{\chi}\right)},
$$

where $\mathrm{K}(\ldots)$ is the complete elliptic integral of the first kind [48], and where

$$
m_{\chi}=\frac{1}{2}\left(1+\frac{\varepsilon}{\left[\varepsilon^{2}+\cos ^{2}(\beta / 2)\right]^{1 / 2}}\right)
$$

and

$$
m_{v}=\frac{1}{2}\left(1-\frac{\varepsilon}{\sin (\beta / 2)}\right)
$$

[1] V. D. Kondratovich and V. N. Ostrovsky, J. Phys. B 23, 3785 (1990).

[2] V. D. Kondratovich and V. N. Ostrovsky, J. Phys. B 17, 1981 (1984); 17, 2011 (1984); 23, 21 (1990).

[3] C. Bordas, Phys. Rev. A 58, 400 (1998).

[4] S. Cohen, P. Kalaitzis, S. Danakas, F. Lépine, and C. Bordas, J. Phys. B 50, 065002 (2017).

[5] T. Barillot, R. Brédy, G. Celep, S. Cohen, I. Compagnon, B. Concina, E. Constant, S. Danakas, P. Kalaitzis, G. Karras, F. Lépine, V. Loriot, A. Marciniak, G. Predelus-Renois, B. Schindler, and C. Bordas, J. Chem. Phys. 147, 013929 (2017).

[6] A. S. Stodolna, F. Lépine, A. Rouzée, S. Cohen, A. Gijsbertsen, J. H. Jungmann-Smith, C. Bordas, and M. J. J. Vrakking, J. Phys. B 50, 164001 (2017).

[7] K. W. Ford and J. A. Wheeler, Ann. Phys. 7, 259 (1959), reissued in 281, 608 (2000).

[8] H. M. Nussenzveig, Diffraction Effects in Semiclassical Scattering (Cambridge University Press, Cambridge, 1992).

[9] J. A. Adam, Phys. Rep. 356, 229 (2002).

[10] C. Nicole, I. Sluimer, F. Rosca-Pruna, M. Warntjes, M. Vrakking, C. Bordas, F. Texier, and F. Robicheaux, Phys. Rev. Lett. 85, 4024 (2000).

[11] C. Bordas, F. Lépine, C. Nicole, and M. J. J. Vrakking, Phys. Rev. A 68, 012709 (2003).

[12] C. Nicole, H. L. Offerhaus, M. J. J. Vrakking, F. Lépine, and C. Bordas, Phys. Rev. Lett. 88, 133001 (2002); F. Lépine, C. Bordas, C. Nicole, and M. J. J. Vrakking, Phys. Rev. A 70, 033417 (2004).

[13] S. Cohen, M. M. Harb, A. Ollagnier, F. Robicheaux, M. J. J. Vrakking, T. Barillot, F. Lépine, and C. Bordas, Phys. Rev. Lett. 110, 183001 (2013); Phys. Rev. A 94, 013414 (2016).
[14] A. S. Stodolna, A. Rouzée, F. Lépine, S. Cohen, F. Robicheaux, A. Gijsbertsen, J. H. Jungmann, C. Bordas, and M. J. J. Vrakking, Phys. Rev. Lett. 110, 213001 (2013).

[15] A. S. Stodolna, F. Lépine, T. Bergeman, F. Robicheaux, A. Gijsbertsen, J. H. Jungmann, C. Bordas, and M. J. J. Vrakking, Phys. Rev. Lett. 113, 103002 (2014).

[16] P. Kalaitzis, S. Danakas, F. Lépine, C. Bordas, and S. Cohen, Phys. Rev. A 97, 053412 (2018).

[17] S. D. López and D. G. Arbó, Phys. Rev. A 100, 023419 (2019).

[18] G. Lantoine and R. P. Russell, Celest. Mech. Dyn. Astr. 109, 333 (2011)

[19] E. Diesen, U. Saalmann, M. Richter, M. Kunitski, R. Dörner, and J. M. Rost, Phys. Rev. Lett. 116, 143006 (2016).

[20] T. F. Gallagher, Rydberg Atoms (Cambridge University Press, Cambridge, 1994), and references therein.

[21] L. B. Zhao and J. B. Delos, Phys. Rev. A 81, 053417 (2010).

[22] R. J. Damburg and V. V. Kolosov, J. Phys. B 9, 3149 (1976).

[23] D. A. Harmin, Phys. Rev. A 24, 2491 (1981).

[24] A.V. Gets and O. I. Tolstikhin, Phys. Rev. A 87, 013419 (2013).

[25] S. Ohgoda, O. I. Tolstikhin, and T. Morishita, Phys. Rev. A 95 , 043417 (2017).

[26] R. R. Freeman, N. P. Economou, G. C. Bjorklund, and K. T. Lu, Phys. Rev. Lett. 41, 1463 (1978); R. R. Freeman and N. P. Economou, Phys. Rev. A 20, 2356 (1979); T. S. Luk, L. DiMauro, T. Bergeman, and H. Metcalf, Phys. Rev. Lett. 47, 83 (1981); W. Sandner, K. A. Safinya, and T. F. Gallagher, Phys. Rev. A 23, 2448 (1981).

[27] V. D. Kondratovich and V. N. Ostrovsky, Zh. Eksp. Teor. Fiz. 79, 395 (1980) [Sov. Phys. JETP 52, 198 (1981)]. 
[28] H. Rottke and K. H. Welge, Phys. Rev. A 33, 301, (1986).

[29] W. L. Glab and M. H. Nayfeh, Phys. Rev. A 31, 530(R) (1985); W. L. Glab, K. Ng, D. Yao, and M. H. Nayfeh, ibid. 31, 3677 (1985).

[30] D. A. Harmin, Phys. Rev. A 26, 2656 (1982).

[31] J. Gao and J. B. Delos, Phys. Rev. A 46, 1455 (1992).

[32] E. Luc-Koenig and A. Bachelier, J. Phys. B 13, 1743 (1980); 13, 1769 (1980).

[33] V. V. Kolosov, Pis'ma Zh. Eksp. Teor. Fiz. 44, 457 (1986) [Sov. Phys. JETP Lett. 44, 588 (1986)].

[34] T. P. Grosdanov, P. S. Krstić, and M. J. Raković, Phys. Lett. A 132, 262 (1988).

[35] S. Watanabe, Phys. Lett. A 126, 107 (1987).

[36] A. K. Kazansky, V. N. Ostrovsky, and D. A. Telnov, J. Phys. B 23, L433 (1990).

[37] A. Alijah, J. Phys. B 25, 5043 (1992).

[38] P. Kalaitzis, S. Danakas, C. Bordas, and S. Cohen, Phys. Rev. A 99, 023428 (2019).

[39] Y. Ralchenko, A. Kramida, and J. Reader, and NIST ASD Team, 2011 NIST Atomic Spectra Database (version 4.1), http: //physics.nist.gov/asd

[40] A. T. J. B. Eppink and D. H. Parker, Rev. Sci. Instrum. 68, 3477 (1997).

[41] M. M. Harb, S. Cohen, E. Papalazarou, F. Lépine, and C. Bordas, Rev. Sci. Instrum. 81, 125111 (2010).

[42] W. E. Cooke and T. F. Gallagher, Phys. Rev. A 17, 1226 (1978); M. G. Littman, M. M. Kash, and D. Kleppner, Phys. Rev. Lett. 41, 103 (1978).

[43] See Supplemental Material at http://link.aps.org/supplemental/ 10.1103/PhysRevA.102.033101 for sequence of experimental near-threshold photoionization microscopy images of $\mathrm{Mg}$ atom as a function of the excited electron's energy.

[44] J. I. Lo, C. C. Chu, H. S. Fung, Y. Y. Lee, and T. S. Yih, Chinese J. Phys. 51, 56 (2013).

[45] W. H. Press, B. P. Flannery, S. A. Teukolsky, and W. T. Vetterling, Numerical Recipes in C: The Art of Scientific Com- puting (Cambridge University Press, Cambridge, 1992); T. H. Cormen, C. E. Leiserson, R. L. Rivest, and C. Stein, Introduction to Algorithms, 2nd ed. (MIT Press and McGraw-Hill, New York, 2001), Chap. 30.

[46] R. B. Blackman and J. W. Tukey, The Measurement of Power Spectra (Dover, New York, 1958).

[47] A. R. P. Rau, J. Phys. B 12, L193 (1979).

[48] M. Abramowitz and I. A. Stegun, Handbook of Mathematical Functions with Formulas, Graphs, and Mathematical Tables (Dover, New York, 1972).

[49] P. Giannakeas, F. Robicheaux, and C. H. Greene, Phys. Rev. A 91, 043424 (2015).

[50] C. Blondel, C. Delsart, and F. Dulieu, Phys. Rev. Lett. 77, 3755 (1996); C. Blondel, C. Delsart, F. Dulieu, and C. Valli, Eur. Phys. J. D 5, 207 (1999).

[51] P. Kalaitzis, D. Spasopoulos, and S. Cohen, Phys. Rev. A 100, 043409 (2019).

[52] B. Broers, J. F. Christian, J. H. Hoogenraad, W. J. van der Zande, H. B. van Linden van denHeuvell, and L. D. Noordam, Phys. Rev. Lett. 71, 344 (1993).

[53] A. Maquet, J. Caillat, and R Taieb, J. Phys. B 47, 204004 (2014); R. Gaillac, M. Vacher, A. Maquet, R. Taïeb, and J. Caillat, Phys. Rev. A 93, 013410 (2016).

[54] M. Schultze, M. Fiess, N. Karpowicz, J. Gagnon, M. Korbman, M. Hofstetter, S. Neppl, A.L. Cavalieri, Y. Komninos, T. Mercouris, C.A. Nicolaides, R. Pazourek, S. Nagele, J. Feist, J. Burgdörfer, A.M. Azzeer, R. Ernstorfer, R. Kienberger, U. Kleineberg, E. Goulielmakis, F. Krausz, and V. S. Yakovlev, Science 328, 1658 (2010).

[55] J.M. Dahlstrom, A. L'Huillier, and A. Maquet, J. Phys. B 45, 183001 (2012).

[56] V. Loriot, A. Marciniak, S. Nandi, G. Karras, M. Hervé, E. Constant, E. Plésiat, A. Palacios, F. Martin, and F. Lépine, J. Phys. Photon. 2, 024003 (2020).

[57] V. V. Beletzky, Essays on the Motion of Celestial Bodies (Mir, Moscow, 1977). 